\title{
NỖ LỰC HỌC TẬP CỦA SINH VIÊN VÀ CÁC HOẠT ĐỘNG GIẢNG DẠY, HỖ TRỌ SINH VIÊN TẠI TRƯỜNG ĐẠI HỌC CÔNG NGHIỆP TP.HCM
}

\author{
NGUYẼ̃N THỊ THU TRANG, NGÔ NGỌC HƯNG, TRÀ̀N ANH DŨNG \\ Truờng Đại học Công nghiệp Thành phố Hồ Chí Minh \\ nguyenthithutrang@iuh.edu.vn,ngongochung@iuh.edu.vn,trananhdung@iuh.edu.vn
}

\begin{abstract}
Tóm tắt. Nghiên cứu này nhằm tìm hiểu nỗ lực học tập của sinh viên và các hoạt động giảng dạy, hỗ trợ sinh viên của trường Đại học Công nghiệp TP. HCM. Dũ liệu được thu thập qua hình thức khảo sát bằng phiếu câu hỏi với sự tham gia của 500 sinh viên. Kết quả nghiên cứu cho thấy (i) nỗ lực học tập của sinh viên chưa cao; (ii) sinh viên đánh giá cao hoạt động giảng dạy của giảng viên và sự hỗ trợ của nhà trường; (iii) thách thức học thuật chưa cao; (iv) mức độ tương tác giữa sinh viên và giảng viên còn hạn chế, chất lượng tương tác của sinh viên với giảng viên và nhân viên chưa cao. Nguyên nhân sinh viên chưa nỗ lực trong học tập có thể do cách kiểm tra đánh giá còn đặt nặng vào đánh giá tổng kết và thách thức học thuật nhà trường đặt ra cho sinh viên chưa cao. Để thúc đẩy sinh viên nỗ lực hơn trong học tập, nhà trường cần chú trọng hơn đến đánh giá quá trình, nâng cao thách thức học thuật và tạo ra một môi trường học tập thân thiện hơn. Nghiên cứu đã phác họa nỗ lực học tập của sinh viên Việt Nam, chỉ ra một số nguyên nhân dẫn đến việc sinh viên chưa nỗ lực nhiều trong học tập và đề xuất một số giải pháp nhằm nâng cao nỗ lực học tập của sinh viên, qua đó nghiên cứu đã có những đóng góp nhất định, về cả lý thuyết và thực tiễn, cho hệ thống tri thức về giáo dục đại học Việt Nam.
\end{abstract}

Từ khóa. Nỗ lực học tập, hoạt động giảng dạy và hỗ trợ sinh viên, thách thức học thuật, tương tác giữa sinh viên và giảng viên, kiểm tra đánh giá.

\section{STUDENT ACADEMIC EFFORT AND TEACHING AND SUPPORTIVE PRACTICES IN INDUSTRIAL UNIVERSITY OF HO CHI MINH CITY}

\begin{abstract}
This study aimed at investigating student academic effort and teaching and supportive practices of Industrial University of Ho Chi Minh city. A survey with the participation of 500 students was employed to collect data. The research findings showed (i) low level of student academic effort; (ii) students' high rating of lecturers' teaching practices and the university's support;(iii) low level of academic challenge (iv) limited interaction between students and lecturers and low quality of students' interaction with lecturers and professional staff. The reason why students did not devote much effort to their study was the university's learning assessment that much emphasized summative assessment and the low level of academic challenge the university presented to students. To promote students' academic effort, the university should focus more on formative assessment, increase levels of academic challenge, and create a more friendly learning environment. The study described Vietnamese students' academic effort, pointed out some reasons for students' low academic effort and suggested some measures for promoting students' academic effort, thereby made certain contribution, both theoretical and practical, to the body of knowledge of Vietnamese higher education.
\end{abstract}

Keywords. Student academic effort, teaching and supportive practices, academic challenge, studentlecturer interaction, learning assessment.

\section{1 ĐặT VÂN ĐỀ}

Nỗ lực học tập (NLHT) của sinh viên được định nghĩa như thời gian và chất lượng của những nỗ lực mà sinh viên dành cho việc học tập cũng như tham gia vào các hoạt động có liên quan đến học tập. Chất lượng của nỗ lực được định nghĩa như sự đầu tư sức lực và trí tuệ của sinh viên để hoàn thành các nhiệm vụ học tập ở mức cao nhất có thể [1]. NLHT luôn được xem là một trong những yếu tố có ảnh hưởng quyết định đến thành tích học tập, sự phát triển trí tuệ và nhân cách của sinh viên. Có chuyên cần, tự giác trong học tập sinh viên mới có thể nắm vững các kiến thức và kỹ năng cần thiết cũng như mới có thể tận 
dụng tốt được các cơ hội học tập, khám phá và phát huy được năng lực của bản thân để thành công trong công việc và cuộc sống sau này [1]. Nhận thức được tầm quan trọng của NLHT của sinh viên, rất nhiều nhà nghiên cứu và quản lý giáo dục đại học trên thế giới đã xem việc thúc đẩy, khuyến khích sinh viên nỗ lực học tập là một trong những biện pháp hàng đầu nhằm nâng cao chất lượng đào tạo. Không ngạc nhiên, khi NLHT của sinh viên cũng như những biện pháp nhà trường sử dụng để nâng cao NLHT của sinh viên đã trở thành tiêu điểm của rẩt nhiều hội thảo hay tranh luận về giáo dục đại học cũng như là đối tượng nghiên cứu của hàng nghìn công trình nghiên cứu khoa học [2]. Bên cạnh đó, khảo sát về NLHT của sinh viên và các hoạt động giảng dạy, hỗ trợ (HĐGD\&HT) của nhà trường đã phát triển mạnh mẽ trong hệ thống giáo dục đại học ở nhiều nơi trên thế giới như Mỹ, Anh, Úc, Trung Quốc ... và được xem như là một thành tố cần thiết trong công tác đảm bảo chất lượng giáo dục đại học [3].

Mặc dù hiếu học luôn được xem là một truyền thống tốt đẹp của người Việt Nam, nhưng đến nay vẫn không có nhiều nghiên cứu về NLHT của sinh viên cũng như những biện pháp nhà trường có thể thực hiện để đẩy mạnh NLHT của sinh viên. Trong bối cảnh hiện nay, khi mà trường Đại học Công nghiệp TP.HCM nói riêng và toàn hệ thống giáo dục đại học ở Việt Nam nói chung đang đứng trước yêu cầu cấp thiết phải đổi mới và cải thiện chất lượng các chương trình đào tạo của mình, NLHT của sinh viên là một khái niệm mới hữu ích về chất lượng cần được quan tâm hơn trong nghiên cứu và các hoạt động thực tiễ̂n của giáo dục đại học ở Việt Nam. Điều đó lại càng có ý nghĩa hơn khi một số nghiên cứu $[4,5]$ đã chỉ ra một trong những nguyên nhân làm giảm sút chất lượng học tập của sinh viên Việt Nam chính là sự thiếu nỗ lực, kém chuyên cần trong học tập của một bộ phận sinh viên. Hiện tượng sinh viên ít nỗ lực trong học tập đã dẫn đến một số hậu quả như một số lượng lớn sinh viên không thể hoàn tất chương trình học đúng thời hạn hay tỷ lệ sinh viên bị cho thôi học tăng đột biến trong những năm gần đây. Ngoài ra, ý thức học tập kém của một bộ phận sinh viên có thể gây ra hậu quả lâu dài và sâu rộng hơn. Do lười học, các sinh viên này có thể sẽ không tích lũy đủ kiến thức và kỹ năng cần thiết cho nghề nghiệp tương lai. Vì vậy, khả năng tìm được việc làm của nhóm sinh viên này sau khi tốt nghiệp tương đối thấp. Theo thống kê mới nhất của Bộ Lao động, Thương binh và Xã hội, trong quý 2/2018, có hơn 126.900 sinh viên tốt nghiệp đã không thể gia nhập vào thị trường lao động.

Thực trạng trên cho thấy đã đến lúc các nhà quản lý và nghiên cứu giáo dục đại học ở Việt Nam cần quan tâm hơn đến NLHT của sinh viên. Nghiên cứu này được thực hiện nhằm tìm hiểu và phác họa các đặc trưng riêng của NLHT của sinh viên Việt Nam cũng như các HĐGD\&HT của trường đại học Việt Nam. Nghiên cứu sẽ kiểm tra xem các đặc trưng riêng này được hình thành và bị tác động ra sao dưới ảnh hưởng của các yếu tố như văn hóa, kinh tế, xã hội ở Việt Nam. Qua đó, kết quả nghiên cứu sẽ có những đóng góp nhất định vào hệ thống tri thức của thế giới về NLHT của sinh viên và các HĐGD\&HT của nhà trường. Nghiên cứu cũng đặt nền móng cho các nghiên cứu chuyên sâu hơn về khái niệm này ở Việt Nam.

\section{TỔNG QUAN TÀI LIỆU}

\subsection{Nỗ lực học tập (NLHT) của sinh viên}

NLHT của sinh viên, như rất nhiều nghiên cứu đã xác nhận, có ảnh hưởng tích cực đến kết quả học tập, kết quả rèn luyện và phát triển nhân cách của sinh viên trong thời gian học đại học.

Nỗ lực được hiểu là "ra sức cố gắng" [6, tr. 736] để thực hiện tốt một công việc nào đó, để hoàn thành một mục tiêu nào đó. Do vậy, NLHT có thể hiểu là sự cố gắng hết sức mình của sinh viên trong học tập nhằm đạt được kết quả học tập tốt nhất có thể. NLHT là sự đầu tư về thời gian, sức lực và tâm trí cho các nhiệm vụ học tập. NLHT thể hiện qua thời gian sinh viên dành cho việc học tập, qua việc thực hiện bài tập đầy đủ trước khi đến lớp, qua sự cố gắng của sinh viên để nắm vững những nội dung khó trong bài, để hoàn thiện các bài tập, tiểu luận .... ở mức tốt nhất, và để có thể đáp ứng được các tiêu chuẩn, yêu cầu của giảng viên, của chương trình học [7]. NLHT thể hiện vai trò chủ đạo của sinh viên trong việc chủ động, tích cực tìm kiếm các cơ hội học tập để nâng cao kiến thức và phát triển các kỹ năng cần thiết cho nghề nghiệp tương lai và cho cuộc sống sau này. 
NLHT chịu ảnh hưởng của một số yếu tố chủ quan, trong đó đáng kể nhất là động cơ học tập và mục đích học tập. Động cơ học tập đóng vai trò như động lực cho các hoạt động học tập diễn ra, thúc đẩy sinh viên thực hiện các hoạt động học tập [8]. Ví dụ, động cơ hoàn thiện tri thức sẽ khuyến khích người học nỗ lực tìm tòi, chiếm lĩnh, và mở rộng tri thức. Nó giúp sinh viên duy trì hứng thú và vượt qua những khó khăn trong học tập [9]. Mục đích học tập định hướng cho những hoạt động học tập của sinh viên. Mục đích học tập giúp sinh viên xác định mình mong muốn đạt được điều gì và mình cần phải làm gì để đạt được điều đó. Mục đích học tập giúp sinh viên kiên trì̀ bền bỉ trong học tập, và thúc đẩy sinh viên phấn đấu, nỗ lực để đạt được kết quả mình mong muốn. Mục đích đặt ra càng cao, sinh viên nỗ lực càng nhiều [10]. Ngoài ra, hứng thú cũng có ảnh hưởng nhất định đến NLHT của sinh viên. Khi có hứng thú với việc học tập, sinh viên sẽ dành nhiều thời gian, công sức hơn để thực hiện các hoạt động học tập.

\subsection{Những hoạt động giảng dạy và hỗ trọ̣ (HĐGD\&HT) của nhà trường}

Mặc dù sinh viên đóng vai trò chủ đạo trong NLHL, nhưng thực tế cho thấy có nhiều sinh viên không tự giác nỗ lực học tập. Trong một nghiên cứu về phong cách học của sinh viên được tiến hành tại trường Đại học Quốc Gia Hà Nội năm 2008, 82,5\% sinh viên cho rằng nỗ lực của người học có ảnh hưởng quyết định đến kết quả học tập. Thế nhưng, chỉ có khoảng 50,3\% sinh viên cho biết các em có cố gắng tự học vài giờ mỗi ngày và chỉ $54,2 \%$ sinh viên cật lực học tập để đạt được mục tiêu học tập của mình [11]. Điều này cho thấy để sinh viên nỗ lực học tập cần phải có sự khuyến khích, thúc đẩy và hỗ trợ của nhà trường. Nhà trường cần phải sử dụng các nguồn tài nguyên (cơ sở vật chất, đội ngũ giảng viên, cán bộ), các chương trình học, các dịch vụ hỗ trợ của mình để tạo ra cơ hội cho sinh viên tham gia nhiều hơn vào các hoạt động học tập [12]. Theo các nhà nghiên cứu Mỹ, các biện pháp mà nhà trường có thể sử dụng để nâng cao nỗ lực học tập của sinh viên bao gồm:

- Tạo ra áp lực học tập cho sinh viên bằng cách nâng cao thách thức học thuật. Thông qua việc đặt ra các tiêu chuẩn và kỳ vọng cao nhưng không quá sức với sinh viên, nhà trường có thể thúc đẩy sinh viên phải nỗ lực tối đa mới có thể đạt được các thành tích cao trong học tập [12]. Để thực hiện được điều đó, toàn bộ chương trình học, phương pháp giảng dạy, các hình thức và nội dung kiểm tra, đánh giá đều cần chú trọng đến việc hình thành và phát triển ở sinh viên các kỹ năng tổng hợp, phân tích, đánh giá thông tin, kỹ năng ứng dụng kiến thức vào thực tế, kỹ năng tích hợp thông tin và chiêm nghiệm các trải nghiệm học tập, khả năng hiểu và sử dụng các thông tin dạng số của sinh viên để đưa ra kết luận để nhận định và đánh giá các lập luận hay vấn đề [13]. Những thách thức về trí tuệ sẽ kích thích sinh viên phải tư duy nhiều hơn, buộc sinh viên phải dành nhiều thời gian hơn cho học tập, đồng thời cũng khơi gợi sự hứng thú của sinh viên đối với học tập. Các nhà nghiên cứu cho rằng nếu không có áp lực từ phía nhà trường và áp lực học tập tự đặt cho bản thân, sinh viên sẽ không cố gắng vượt qua giới hạn của bản thân để có thể nâng cao kiến thức hoặc chuyên tâm lâu dài với việc học tập [14]. Tuy nhiên, một số nhà nghiên cứu [12], [15] đã khuyển cáo rằng để đạt được kết quả như mong muốn, áp lực học tập cần phải đi đôi với sự hỗ trợ sinh viên trong học tập. Sự hỗ trợ thể hiện qua các hoạt động định hướng, tư vấn, các trung tâm bồi dưỡng các kỹ năng học thuật cần thiết nhưng còn thiếu cho sinh viên cũng như giúp đỡ cho các sinh viên gặp khó khăn trong học tập;

- Hoạt động giảng dạy hiệu quả của giảng viên thể hiện qua việc chuẩn bị bài kỹ lưỡng, trình bày bài giảng logic, hướng dẫn rõ ràng, giải thích dễ hiểu, cũng như đưa ra các phản hồi chi tiết, kịp thời, thường xuyên về các bài tập, bài kiểm tra của sinh viên [13]. Những hoạt động giảng dạy này, theo các nhà nghiên cứu Mỹ, sẽ giúp sinh viên tiếp thu kiến thức hiệu quả hơn, qua đó, có tác động tích cực đến kết quả học tập của sinh viên;

- Tạo điều kiện để sinh viên có thể hợp tác với các bạn học trong các hoạt động học tập khác nhau (giải thích bài học, chuẩn bị các bài thi, làm việc nhóm trong các đồ án hay tiểu luận) cũng như có thể tiếp cận và tham khảo ý kiến của giảng viên về các vấn đề liên quan đến học tập và hướng nghiệp (thảo luận với giảng viên về các chủ đề môn học, kết quả học tập của mình, kế hoạch nghề nghiệp trong tương lai) hay tham gia cùng giảng viên trong các hoạt động nghiên cứu và các hoạt động ngoại khóa [13]. Tương tác với bạn bè và giảng viên sẽ giúp sinh viên mở rộng kiến thức và 
phát triển kỹ năng, giúp sinh viên hài lòng hơn với các trải nghiệm học tập tại trường và giúp làm tăng tình cảm gắn bó của sinh viên với nhà trường [12];

- Tạo ra một môi trường hỗ trợ thông qua các chính sách, các trung tâm và dịch vụ giúp đỡ sinh viên trong cả học tập và cuộc sống. Nhà trường cần thể hiện sự quan tâm đối với sinh viên, thường xuyên tổ chức các sự kiện văn hóa nhằm nâng cao đời sống tinh thần của sinh viên, và tạo ra bầu không khí thân thiện giữa sinh viên với các thành viên khác trong nhà trường (bạn học, giảng viên, cố vấn học tập, cán bộ, nhân viên). Nhà trường cần xem sự chăm sóc và giúp đỡ sinh viên đạt thành quả trong học tập và phát triến nhân cách là một trong những nhiệm vụ quan trọng hàng đầu của mình [17]. Một môi trường hỗ trợ sẽ tạo cho sinh viên sự hài lòng với nhà trường, giúp sinh viên hòa nhập, gắn bó với nhà trường, và cũng góp phần đáng kể vào thành tích học tập và rèn luyện của sinh viên [17].

Nhiều nghiên cứu sâu rộng được tiến hành ở các trường đại học ở Mỹ, Anh, Úc đã chỉ ra được ảnh hưởng tích cực của NLHT của sinh viên và các HĐGD\&HT của nhà trường đối với điểm số, sự phát triển các kỹ năng nhận thức và tư đuy, các kỹ năng mềm của sinh viên, sự kiên trì trong học tập, sự hài lòng của sinh viên đối với trải nghiệm học tập ở nhà trường, sự phát triển về nhân cách và đạo đức và khả năng học tập suốt đời của sinh viên [17, 18, 19, 20].

Mặc dù không ai phủ nhận ảnh hưởng của NLHT của sinh viên, các HĐGD\&HT của nhà trường đối với kết quả học tập, rèn luyện và sự phát triển nhân cách của sinh viên, các nhà nghiên cứu vẫn còn tranh cãi khá nhiều về mức độ tương quan giữa các đại lượng này. Một số nhà nghiên cứu $[19,20]$ cho rằng NLHT của sinh viên và HĐGD\&HT của nhà trường có ảnh hưởng tích cực và có ý nghĩa về mặt thống kê với kết quả học tập và rèn luyện của sinh viên. Trong khi đó, một số nghiên cứu khác $[19,21]$ lại xác định mức độ ảnh hưởng của NLHT của sinh viên, các HĐGD\&HT của nhà trường đối với thành tích học tập và sự phát triển của sinh viên là không đáng kể. Ngoài ra các nghiên cứu cũng cho thấy mối quan hệ giữa các đại lượng này còn chịu tác động của nhiều yếu tố khác như đặc điểm của sinh viên, của nhà trường, gia đình, bạn bè và xã hội $[2,18,20]$. Các kết quả nghiên cứu trên đã phản ánh quan điểm đa chiều của các nhà nghiên cứu về mức độ tác động của NLHT của sinh viên, các HĐGD\&HT của nhà trường đối với kết quả học tập và rèn luyện của sinh viên. Tuy nhiên, các nhà nghiên cứu đều thống nhất nhìn nhận NLHT của sinh viên cùng với HĐGD\&HT của nhà trường là một trong những chỉ số dự báo tốt nhất về thành tích học tập và sự phát triển nhân cách của sinh viên.

\section{PHƯƠNG PHÁP NGHIÊN CÚU}

Nghiên cứu được tiến hành vào tháng 4/2018, tại trường đại học Công nghiệp TP. HCM, với sự tham gia của các sinh viên đại học hệ chính quy năm thứ nhất và năm thứ tư đang theo học tại trường. Năm thứ nhất và năm thứ tư được xem là hai cột mốc quan trọng nhất trong toàn bộ thời gian học đại học. Năm thứ nhất đặt nền móng cho toàn bộ quá trình học tập. Đây là thời gian sinh viên phải làm quen với môi trường sinh hoạt, học tập mới, phương pháp học tập mới, hình thành những kỹ năng học tập mới. Những điều này có thể tạo nên một số áp lực đối với sinh viên. Những áp lực này có thể là một trong những nguyên nhân dẫn đến tình trạng bỏ học cao ở sinh viên năm thứ nhất. Chính vì vậy, sinh viên năm thứ nhất sẽ cần đến sự hướng dẫn, hỗ trợ của nhà trường nhiều nhất. Thông tin về sinh viên năm thứ nhất sẽ giúp nhà trường hiểu rõ hơn những khó khăn sinh viên có thể gặp phải để có những biện pháp hỗ trợ cần thiết giúp sinh viên có thể thích nghi và thành công trong những năm học kế tiếp. Năm thứ tư là thời điểm kết thúc của quá trình học. Do sinh viên năm thứ tư có thời gian học tập tại trường dài nhất, họ có thể đưa ra những đánh giá toàn diện và chính xác về trải nghiệm học tập tại trường. Hình thức khảo sát bằng phiếu câu hỏi được sử dụng để thu thập dữ liệu. 500 sinh viên tham gia khảo sát được chọn lựa ngẫu nhiên theo cụm dựa trên đơn vị lớp học.

Phiếu câu hỏi khảo sát của nghiên cứu được xây dựng dựa trên bảng câu hỏi College Survey Report (CSR) được NSSE sử dụng để đánh giá NLHT của sinh viên và các HĐGD\&HT của nhà trường của Mỹ. Nhà nghiên cứu có thực hiện một số điều chỉnh trên phiếu khảo sát để phù hợp với điều kiện và môi trường giáo dục ở Việt Nam. Phiếu câu hỏi có 22 câu hỏi lớn, 98 mục hỏi, trong đó 43 mục hỏi được 
sử dụng để xây dựng 10 chỉ báo đo lường đánh giá của sinh viên về NLHT của họ cũng như các HĐGD\&HT của nhà trường. Thang đo Likert ( 4 điểm, 5 điểm, 7 và 8 điểm) được sử dụng để đo lường các đánh giá của sinh viên. Tên các chỉ báo, số mục hỏi, nội dung của chỉ báo được trình bày chi tiết trong bảng 1 .

Bảng 1: Các chỉ báo NLHT của sinh viên và các HĐGD\&HT của nhà trường

\begin{tabular}{|c|c|c|c|}
\hline Chủ đề & Chỉ bảo & $\begin{array}{l}\text { Số } \\
\text { mục } \\
\text { hỏi }\end{array}$ & Dữ liệu thu thập \\
\hline \multirow{4}{*}{$\begin{array}{l}\text { Thách thức } \\
\text { học thuật }\end{array}$} & $\begin{array}{l}\text { Phát triển tư duy } \\
\text { bậc cao }\end{array}$ & 4 & $\begin{array}{l}\text { Mức độ chú trọng của chương trình đào tạo đến việc } \\
\text { phát triển các hoạt động tư duy bậc cao: phân tích, đánh } \\
\text { giá, vận dụng kiến thức và tạo ra kiến thức mới. }\end{array}$ \\
\hline & $\begin{array}{l}\text { Học tập thông qua } \\
\text { tích hợp và chiêm } \\
\text { nghiệm }\end{array}$ & 6 & $\begin{array}{l}\text { Mức độ sinh viên thường xuyên tích hợp kiến thức từ } \\
\text { nhiều nguồn tài liệu, môn học khác nhau trong các bài } \\
\text { tiểu luận, kiểm tra; liên kết kiến thức đườc học với kinh } \\
\text { nghiệm bản thân; phân tích điểm mạnh, yếu trong lập } \\
\text { luận của bản thân, hiểu quan điểm của người khác ... }\end{array}$ \\
\hline & Chiến lược học tập & 3 & $\begin{array}{l}\text { Mức độ sinh viên thường xuyên xác định thông tin } \\
\text { chính, xem lại ghi chép, tóm tắt bài học ... }\end{array}$ \\
\hline & $\begin{array}{l}\text { Lập luận định } \\
\text { lượng }\end{array}$ & 3 & $\begin{array}{l}\text { Mức độ sinh viên thường xuyên sử dụng số liệu để đưa } \\
\text { ra kêt luận, phân tích và đánh giá thông tin. }\end{array}$ \\
\hline $\begin{array}{l}\text { Hợp tác trong } \\
\text { học tâp }\end{array}$ & $\begin{array}{l}\text { Hợp tác trong học } \\
\text { tập }\end{array}$ & 4 & $\begin{array}{l}\text { Mức độ sinh viên thường xuyên hợp tác với bạn học khi } \\
\text { tìm hiểu hay ôn tập bài học, làm đồ án, tiểu luận. }\end{array}$ \\
\hline \multirow{2}{*}{$\begin{array}{l}\text { Trải nghiệm } \\
\text { cùng giảng } \\
\text { viên }\end{array}$} & $\begin{array}{l}\text { Tương tác giữa } \\
\text { sinh viên và giảng } \\
\text { viên }\end{array}$ & 5 & $\begin{array}{l}\text { Mức độ sinh viên thường xuyên trao đổi với giảng viên } \\
\text { về bài học trong và ngoài lớp học, về kêt quả học tập, về } \\
\text { dự định nghề nghiệp và cùng tham gia với giảng viên } \\
\text { trong các hoạt động ngoại khóa. }\end{array}$ \\
\hline & $\begin{array}{l}\text { Hoạt động giảng } \\
\text { dạy hiệu quả }\end{array}$ & 5 & $\begin{array}{l}\text { Mức độ giảng viên sử dụng các hoạt động giảng dạy } \\
\text { (trình bày, nêu ví dụ, nhận xét, phản hồi bài tập, bài } \\
\text { kiểm tra của sinh viên...). }\end{array}$ \\
\hline \multirow{2}{*}{$\begin{array}{l}\text { Môi trường } \\
\text { học tập }\end{array}$} & $\begin{array}{l}\text { Chất lượng tương } \\
\text { tác }\end{array}$ & 4 & $\begin{array}{l}\text { Sinh viên đánh giá chất lượng mối quan hệ của mình với } \\
\text { bạn học, với giảng viên, cố vấn học tập và nhân viên } \\
\text { phòng ban. }\end{array}$ \\
\hline & $\begin{array}{l}\text { Sự hỗ trợ của nhà } \\
\text { trường }\end{array}$ & 4 & $\begin{array}{l}\text { Mức độ chú trơng của nhà trường đối với các hoạt động } \\
\text { hồ trợ sinh viên trong học tập và trong cuộc sống. }\end{array}$ \\
\hline $\begin{array}{l}\text { Nỗ lực học tập } \\
\text { của sinh viên }\end{array}$ & $\begin{array}{l}\text { Nỗ lực học tập của } \\
\text { sinh viên }\end{array}$ & 5 & $\begin{array}{l}\text { Thời gian sinh viên dành cho việc chuẩn bị bài. Mức độ } \\
\text { sinh viên thường xuyên: chuẩn bị bài trước khi đến lớp; } \\
\text { cố gắng nắm vững các nội dung khó trong bài, hoàn } \\
\text { thiện ở mức tốt nhất các bài tập, tiểu luận; cố gắng hết } \\
\text { sức để có thể đáp ứng được các yêu cầu, tiêu chuẩn của } \\
\text { giảng viên. }\end{array}$ \\
\hline
\end{tabular}

Trong 10 chỉ báo, 9 chỉ báo đầu tiên được xây dựng dựa trên chỉ báo của khảo sát NSSE của Mỹ, chỉ báo Nỗ lực học tập của sinh viên được nhà nghiên cứu xây dựng dựa trên tài liệu và các nghiên cứu trước đó ở Mỹ và ở UUc về NLHT của sinh viên. Hệ số Cronbach's alpha được sử dụng để đánh giá độ tin cậy của 10 thang đo. Trừ chỉ báo Chiến lược học tập có hệ số Cronbach's alpha 0,65 , ở các chỉ báo còn lại hệ số này dao động từ 0,70 cho đến 0,85 . Điều này cho thấy các thang đo được sử dụng trong nghiên cứu này đã đạt được độ tin cậy cần thiết. 
Bảng 2: Hệ số Cronbach’s alpha của các chỉ báo

\section{Các chỉ báo}

\begin{tabular}{lcccccccccc}
\hline & TDBC & TH\&CN & CLHT & LLĐL & HTc & $\begin{array}{c}\text { TTGV- } \\
\text { SV }\end{array}$ & GDHQ & CLTT & HTr & NLHT \\
\hline $\begin{array}{l}\text { Cronbach's } \\
\text { alpha } \alpha\end{array}$ & 0,76 & 0,77 & 0,65 & 0,76 & 0,70 & 0,80 & 0,85 & 0,84 & 0,77 & 0,70 \\
\hline
\end{tabular}

Chú thích: $T D B C=$ Phát triển tu duy bậc cao; TH\&CN=Học tập thông qua tích hợp và chiêm nghiệm, CLHT =

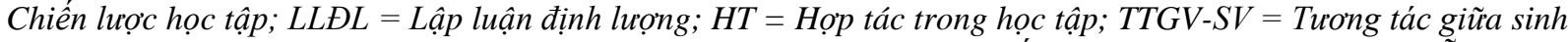
viên và giảng viên; $G D H Q=$ Hoạt động giảng dạy hiệu quả; CLTT = Chất luợng tuơng tác; Htr = Sụ hỗ trợ của nhà trường; NLHT = Nố lực họ tập của sinh viên

Dữ liệu thu thập từ phiếu khảo sát được xử lý bằng phần mềm thống kê SPSS 22.0. Thống kê mô tả (giá trị trung bình, độ lệch chuẩn $(\mathrm{SD})$, tỷ lệ phần trăm) được sử dụng để phân tích các đặc điểm của mẫu khảo sát và mô tả các chỉ báo đo lường NLHT của sinh viên, các HĐGD\&HT của nhà trường. Ngoài ra, nghiên cứu còn sử dụng phép tính thống kê t-test để tìm hiểu sự khác biệt trong nỗ lực học tập và sự hỗ trợ của nhà trường giữa sinh viên nam và nữ, sinh viên năm thứ nhất và năm thứ tư, sinh viên theo học khối ngành kỹ thuật và khối ngành kinh tế.

\section{KẾT QUẢ NGHIÊN CÚU VÀ THẢO LUẬN}

\section{1 Đặc điểm của mẫu khảo sát}

Tổng số sinh viên tham gia vào khảo sát bao gồm 500 sinh viên hệ đại học chính quy.

Về giới tính, mẫu khảo sát có 155 sinh viên nam (chiếm $31 \%$ tổng số sinh viên tham gia khảo sát) và 345 sinh viên nữ (chiếm 69\%). Về chuyên ngành học, mẫu khảo sát phân bố ở hầu hết các chuyên ngành đào tạo tại trường. Trong nghiên cứu này, mẫu khảo sát được chia thành hai nhóm lớn: nhóm kỹ thuật (điện tử, công nghệ thông tin, cơ khí ...) và nhóm kinh tế (kế toán, quản trị kinh doanh, thương mại du lịch ...). Nhóm kỹ thuật bao gồm 276 sinh viên (55,2\%), nhóm kinh tế có 224 sinh viên $(44,8 \%)$. Về năm học, mẫu khảo sát có 250 sinh viên năm thứ nhất $(50 \%)$ và 250 sinh viên năm thứ tư $(50 \%)$.

Bảng 3: Đặc điểm mẫu khảo sát

\begin{tabular}{llcc}
\hline & Đặc điểm & Số lượng & Tỷ lệ (\%) \\
\hline \multirow{2}{*}{ Giới tính } & Nam & 155 & 31 \\
& Nữ & 345 & 69 \\
\multirow{3}{*}{ Năm học } & Thứ nhất & 250 & 50 \\
& Thứ tư & 250 & 50 \\
\multirow{2}{*}{ Ngành học } & Kỹ thuật & 276 & 55,2 \\
& Kinh tế & 224 & 44,8 \\
\hline
\end{tabular}

\subsection{Các chỉ báo}

Các chỉ báo được tính trên thang điểm 60 vì hai lý do: (i) các chỉ báo được đo lường bằng các thang đo tổng Likert với nhiều mức độ khác nhau, việc đưa về cùng một thang điểm sẽ giúp việc so sánh các chỉ báo dễ dàng hơn; (ii) trong khảo sát NSSE các chỉ báo cũng được tính trên thang điểm 60 , việc chuyển đổi các chỉ báo sang thang điểm 60 sẽ giúp cho việc so sánh kết quả của nghiên cứu này với kết quả nghiên cứu của NSSE thuận tiện hơn. 
Bảng 4: Trung bình (means) và độ lệch chuẩn $(\mathrm{SD})$ của các chỉ báo

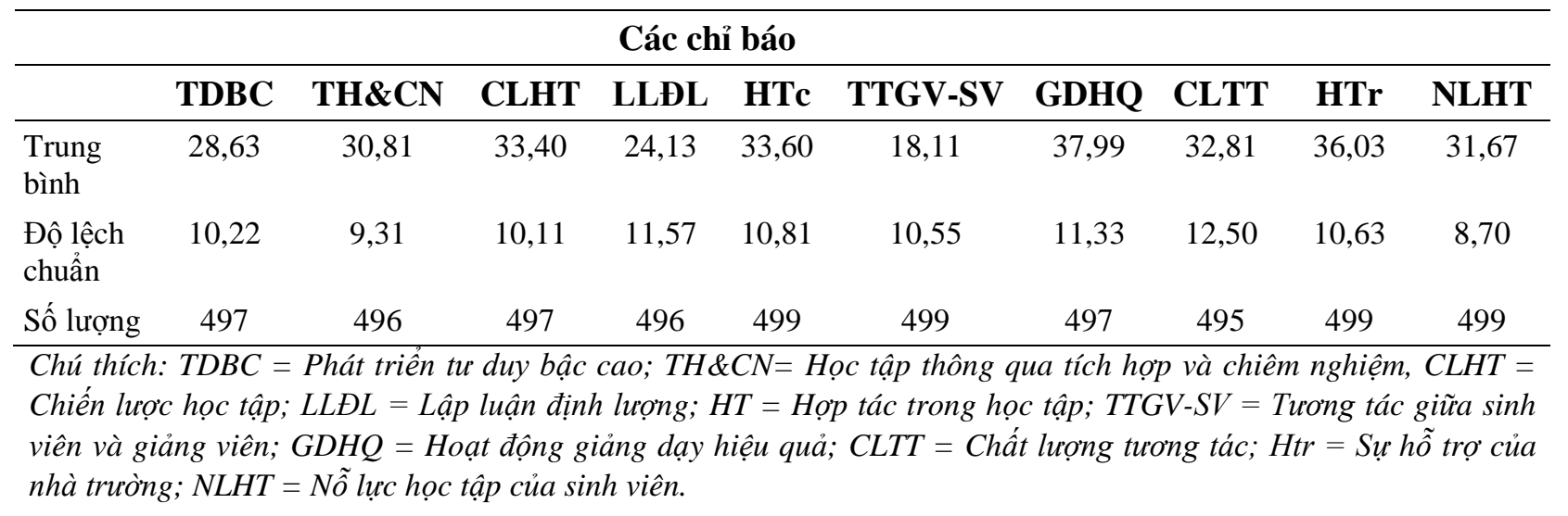

Xét tổng thể, các chỉ báo NLHT của sinh viên, các HĐGD\&HT của nhà trường chỉ ở mức trung bình thấp. Ngoại trừ chỉ báo Hoạt động giảng dạy hiệu quả $(\mathrm{M}=37,99)$ và Sự hỗ trợ của nhà trường $(\mathrm{M}=36,03$ ), giá trị trung bình của $5 / 10$ chỉ báo chỉ đạt từ 30,81 đến 33,60 . Đáng chú ý, $3 / 10$ chỉ báo (Phát triển tư duy bậc cao, Tương tác giữa giảng viên và sinh viên, Lập luận định lượng) có giá trị trung bình thấp hơn 30. Giá trị trung bình này cũng thấp hơn nhiều so với giá trị trung bình của các chỉ báo tương ứng trong khảo sát sinh viên Mỹ NSSE năm 2018 (9 chỉ báo), biến thiên từ 21,5 (Tương tác giữa sinh viên và giảng viên) cho đến 41,8 (Chất lượng tương tác). Khoảng cách giá trị trung bình cao nhất $(\mathrm{M}=$ $37,99)$ và giá trị trung bình thấp nhất $(M=18,11)$ khá rộng, lên đến 19,88 . Phần thảo luận kết quả của từng chỉ báo theo chủ đề sẽ được trình bày chi tiết dưới đây.

Bảng 5: Giá trị trung bình các chỉ báo của Việt Nam và Mỹ

\begin{tabular}{lcc}
\hline \multirow{2}{*}{ Các chỉ báo } & \multicolumn{2}{c}{ Giá trị trung bình (Means) } \\
\cline { 2 - 3 } & Việt Nam & Mỹ \\
\hline Phát triển tư duy bậc cao & 28,63 & 38,4 \\
Học tập thông qua tích hợp và chiêm nghiệm & 30,81 & 35,4 \\
Chiến lược học tập & 33,40 & 38,3 \\
Lập luận định lượng & 24,13 & 27,1 \\
Hợp tác trong học tập & 33,60 & 33,8 \\
Tương tác giữa sinh viên và giảng viên & 18,11 & 21,5 \\
Hoạt động giảng dạy hiệu quả & 37,99 & 38,6 \\
Chất lượng tương tác & 32,81 & 41,8 \\
Sự hỗ trợ của nhà trường & 36,03 & 36,6 \\
\hline Ś́
\end{tabular}

Nguồn: Số liệu điều tra trục tiếp, 2018; [22]

\subsection{Các hoạt động giảng dạy và hỗ trợ của nhà trường}

Các hoạt động giảng dạy và hỗ trợ sinh viên của nhà trường được chia thành 4 chủ đề chính: Thách thức học thuật, Hợp tác trong học tập, Trải nghiệm cùng giảng viên và Môi trường học tập.

\subsubsection{Thách thức học thuật}

Ngoại trừ chỉ báo Chiến lược học tập $(\mathrm{M}=33,40)$, giá trị trung bình của các chỉ báo không cao, đặc biệt chỉ báo Lập luận định lượng chỉ đạt giá trị trung bình 24,13. Các giá trị trung bình này chênh lệch đáng kể so với giá trị trung bình của các chỉ báo tương ứng trong khảo sát NSSE 2018 của Mỹ (biến thiên từ 27,1 đến 38,4). Mức chênh lệch cao nhất thuộc về 2 chỉ báo Phát triển tư duy bậc cao và Học tập thông qua tích hợp và chiêm nghiệm (Việt Nam: 28,63 và 30,80 so với Mỹ: 38,40 và 35,40). Ví dụ, trong khi 
hơn $70 \%$ sinh viên Mỹ được khảo sát cho rằng kiểm tra đánh giá chú trọng nhiều đến phát triển tư duy bậc cao, chỉ có khoảng $50 \%$ sinh viên Việt Nam được khảo sát có cùng ý kiến. Riêng với yêu cầu ứng dụng kiến thức đã học vào các vấn đề thực tế, chỉ có $32 \%$ sinh viên Việt Nam cho rằng các bài kiểm tra có chú trọng đến hoạt động tư duy bậc cao này. Tương tự, có đến $70 \%$ sinh viên Mỹ thừa nhận họ phải thường xuyên tích hợp kiến thức từ nhiều môn học khác nhau khi làm bài kiểm tra hay viết tiểu luận, trong khi tỷ lệ này ở Việt Nam chỉ ở mức 36\%. Chỉ khoảng $56 \%$ sinh viên Việt Nam thường xuyên liên kết các kiến thức được học với các kinh nghiệm và kiến thức đã có trước đó. Tỷ lệ này khá chênh lệch với tỷ lệ 84\% sinh viên Mỹ thường xuyên thực hiện hoạt động này.

Kết quả khảo sát đã cho thấy thách thức học thuật nhà trường đặt ra cho sinh viên chưa cao. Nhà trường cần phải chú trọng hơn đến việc phát triển tư duy bậc cao của sinh viên hay khuyến khích sinh viên học tập thông qua tích hợp và chiêm nghiệm. Những hoạt động này, theo nhiều nhà nghiên cứu [14, 23] sẽ đặt ra cho sinh viên những thách thức trí tuệ, buộc họ phải dành nhiều thời gian hơn cho học tập, phải tư duy nhiều hơn, tìm tòi, khám phá, sáng tạo nhiều hơn, tương tác nhiều hơn với bạn học và giảng viên. Từ những nỗ lực này, sinh viên sẽ phát huy được các năng lực của mình và có thể đạt được mức phát triển cao nhất của bản thân.

\subsubsection{Họp tác trong học tập}

Việc sinh viên hợp tác với nhau trong học tập như giúp nhau hiểu bài, chia sẻ thông tin, cùng nhau thực hiện bài tập nhóm, đồ án, cùng ôn thi ..., theo một số nhà nghiên cứu [23], sẽ tạo động lực học tập cho sinh viên, giúp sinh viên phát triển kỹ năng giao tiếp và giải quyết vấn đề cũng như tăng thêm sự tương tác tích cực giữa sinh viên với nhau. Giá trị trung bình của chỉ báo này không cao nhưng ở mức chấp nhận được $(\mathrm{M}=33,60)$ và không có chênh lệch đáng kể với dữ liệu thu được từ NSSE $2018(\mathrm{M}=$ 33,8). Có gần $70 \%$ sinh viên Việt Nam được khảo sát thường xuyên thực hiện đồ án, tiểu luận cùng các bạn và $68,7 \%$ sinh viên thường xuyên nhờ bạn giải thích tài liệu môn học. Tín hiệu tích cực này có thể là kết quả của việc nhà trường tăng cường áp dụng các phương pháp giảng dạy mới như làm việc nhóm, Project Based Learning (PBL)... Điều này đã tạo cho sinh viên có cơ hội hợp tác nhiều hơn với các bạn trong học tập.

\subsubsection{Trải nghiệm cùng giảng viên}

Chủ đề trải nghiệm cùng giảng viên bao gồm hai khía cạnh: đánh giá của sinh viên về các hoạt động giảng dạy của giảng viên và mức độ tương tác giữa sinh viên và giảng viên. Trong khi sinh viên đánh giá cao hoạt động giảng dạy của giảng viên $(\mathrm{M}=37,99)$, chỉ báo Tương tác giữa sinh viên và giảng viên có giá trị trung bình thấp nhất $(\mathrm{M}=18,11)$ trong số 10 chỉ báo. Dữ liệu cho thấy có hơn $3 / 4$ sinh viên tham gia khảo sát cho rằng giảng viên của họ có giải thích rõ mục tiêu, yêu cầu của môn học, thực hiện bài giảng có hệ thống, logic, và có đưa ra các ví dụ minh họa để bài giảng sinh động, dễ hiểu (tỷ lệ tương ứng: $76,9 \%, 82,9 \%$ và $76,1 \%$ ). Kết quả trên cho thấy nhà trường có một đội ngũ giảng viên có chất lượng, giảng dạy hiệu quả. Ngoài ra, có gần $2 / 3$ sinh viên được hỏi cho rằng giảng viên có thực hiện việc phản hồi, nhận xét bài làm, bài kiểm tra của sinh viên ở mức độ cao. Tỷ lệ này tăng nhiều so với tỷ lệ thu được ở một cuộc khảo sát tương tự có trường này tham gia được thực hiện vào năm 2013 [24]. Trong cuộc khảo sát đó chỉ có khoảng $48 \%$ sinh viên cho rằng họ nhận được phản hồi của giảng viên với bài làm của họ. Đây là một sự thay đổi đáng khích lệ. Phản hồi của giảng viên sẽ giúp sinh viên nhận ra các điểm còn yếu trong bài làm của mình để có thể cải thiện và đạt thành tích tốt hơn.

Trái ngược với kết quả tích cực nêu trên, đa số sinh viên tham gia khảo sát cho biết họ không thường xuyên tương tác với giảng viên. Ví dụ, chỉ có $13 \%$ sinh viên có trao đổi với giảng viên về dự định nghề nghiệp của họ, $14 \%$ có trao đổi với giảng viên về kết quả học tập. Trong khi tỷ lệ tương ứng ở Mỹ là $44 \%$ và $35 \%$. Các tương tác giữa sinh viên và giảng viên chủ yếu diền ra ở lớp học khi sinh viên trao đổi với giảng viên về nội dung bài học. Tuy nhiên, tỷ lệ này cũng chỉ dừng ở mức $26,7 \%$. Nguyên nhân của mức độ tương tác thấp giữa giảng viên và sinh viên có thể là do giảng viên chỉ ở trường khi có tiết giảng 
dạy, sinh viên ít có cơ hội gặp giảng viên bên ngoài lớp học. Ngoài ra, có thể mối quan hệ theo tôn ti, cấp bậc giữa giảng viên và sinh viên đã làm hạn chế sự tương tác giữa họ. Các khảo sát được thực hiện ở Trung Quốc và Hàn Quốc, hai nước chịu ảnh hưởng của đạo Khổng như Việt Nam, cũng đã ghi nhận mức độ tương tác khá thấp giữa sinh viên và giảng viên [25,26]. Theo một số nhà nghiên cứu [16], mối tương tác giữa sinh viên và giảng viên có ảnh hưởng tích cực đối với thành tích học tập, việc chọn lựa nghề nghiệp, sự phát triển các kỹ năng tư duy, trí tuệ, nhân cách và đạo đức của sinh viên. Do vậy, nhà trường cũng nên lưu ý tạo điều kiện để thúc đẩy sự tương tác giữa sinh viên và giảng viên.

\subsubsection{Môi trường học tập}

Đánh giá của sinh viên về 2 khía cạnh trong chủ đề này: Chất lượng tương tác và Sự hỗ trợ của nhà trường tương phản với nhau. Dữ liệu cho thấy đa số sinh viên cho rằng nhà trường hỗ trợ nhiều cho họ trong học tập, trong việc phát triển các mối giao tiếp xã hội trong nhà trường, hỗ trợ về mặt tài chính và một số vấn đề trong đời sống (tỷ lệ tương ứng $60,9 \%, 70,9 \%$ và $57,1 \%$ ). Điều này chứng tỏ nhà trường đã có sự chăm lo, quan tâm đáng kể đến sinh viên. Tuy nhiên, khi được yêu cầu đánh giá về chất lượng mối quan hệ với các thành viên trong trường bao gồm bạn học, giảng viên, cố vấn học tập và các nhân viên, câu trả lời của sinh viên không được tích cực như vậy. Chất lượng tương tác được đánh giá trên thang điểm từ 1 đến 7 , trong đó 1 là mức điểm thấp nhất chỉ mối quan hệ tồi tệ, 7 chỉ mối quan hệ ở mức tuyệt vời. Tỷ lệ sinh viên cho rằng họ có mối quan hệ tốt (điểm 6,7$)$ với bạn học ở mức $44,2 \%$. Tỷ lệ này giảm đáng kể khi sinh viên đánh giá mối quan hệ giữa họ và giảng viên $(17,2 \%)$, cố vấn học tập $(19,3 \%)$ và nhân viên phòng ban $(12,6 \%)$. Tỷ lệ này khá thấp so với đánh giá của sinh viên Mỹ về mối quan hệ của họ với giảng viên $(56 \%)$, cố vấn học tập $(51 \%)$, và nhân viên $(41 \%)$. Điều này đặt ra yêu cầu cần cải thiện hơn nữa chất lượng, thái độ của các thành viên trong trường nhằm tạo ra một môi trường học tập thân thiện hơn cho sinh viên. Môi trường học tập thân thiện sẽ làm cho sinh viên hài lòng và gắn bó hơn với nhà trường và có ảnh hưởng tích cực đến kết quả học tập của sinh viên [16].

\subsection{Nỗ lực học tập của sinh viên}

Phân tích đánh giá của sinh viên cho từng hoạt động liên quan đến chỉ báo này cho thấy đa số sinh viên thường xuyên cố gắng nắm vững những nội dụng khó trong bài $(71,1 \%)$ hay cố gắng hoàn thiện các bài tập, tiểu luận $(85 \%)$. Tuy nhiên, có hơn phân nửa sinh viên thừa nhận họ không thường xuyên chuẩn bị bài khi đến lớp $(56 \%)$ và không thường xuyên học tập chăm chỉ để đáp ứng được các yêu cầu của giảng viên $(59,3 \%)$. Đặc biệt, số liệu cho thây sinh viên chỉ dành 12 tiếng/tuần để chuẩn bị bài ở nhà. Số giờ dành cho việc tự học này thấp hơn nhiều so với yêu cầu của chương trình đào tạo theo học chế tín chí. Theo quy định một giờ học trên lớp đòi hỏi sinh viên phải bỏ ra 2 giờ để chuẩn bị bài, tự học ở nhà, Bình quân một tuần sinh viên sẽ có ít nhất 10 giờ học trên lớp, khi đó số giờ tự học tối thiểu của sinh viên phải lên đến 20 giờ/tuần. Những số liệu trên cho thấy sinh viên của trường chưa nỗ lực nhiều trong học tập. Điều này phù hợp với những than phiền về việc sinh viên thiếu chuyên cần, còn lơ là trong học tập được nêu trong các nghiên cứu trước đó hoặc trên báo chí trong thời gian gần đây $[4,5,24]$. Tình trạng này có thể do các nguyên nhân sau:

- Sự chú trọng đến đánh giá tổng kết, xem nhẹ đánh giá quá trình. Tại trường Đại học Công nghiệp TP.HCM, điểm đánh giá thường kỳ chỉ chiếm tỷ trọng có $20 \%$ điểm tổng kết môn học, trong khi điểm thi (giữa kỳ và cuối kỳ) chiếm đến $80 \%$. Sự chênh lệch trong đánh giá này dẫn đến việc sinh viên có thể lơ là học tập trong cả học kỳ và chỉ cần tập trung học vào những kỳ thi cũng có thể qua môn. Có đến $65,5 \%$ sinh viên tham gia khảo sát cho rằng các kỳ thi mới là tác nhân chính buộc họ phải nỗ lực học tập ở mức cao nhất. Hiện tượng này cũng đã được các nhà nghiên cứu Trung Quốc [27] ghi nhận khi khảo sát nỗ lực học tập của sinh viên Trung Quốc.

- Thách thức học thuật chưa cao. Theo một số nhà nghiên cứu, thách thức học thuật sẽ đặt ra các thử thách thúc đây sinh viên phải nỗ lực học tập để nâng cao kiến thức, để đáp ứng được yêu cầu của môn học, khóa học, giúp họ đạt đến mức độ phát triển cao nhất có thể của bản thân. Ngược lại, nếu thách thức chưa đủ cao, một số sinh viên, mặc dù chây lười, không cần học tập hết sức vẫn có khả năng hoàn thành môn học, khóa học. Theo phân tích số liệu ở trên thách thức học thuật của trường chưa cao, và đây có thể là nguyên nhân dẫn đến sự thiếu chuyên cần của sinh viên. 


\subsection{Sự khác biệt trong các chỉ báo giữa các nhóm sinh viên}

Phép tính thống kê t-test được sử dụng để tìm hiểu sự khác biệt trong NLHT, HĐGD\&HT của nhà trường giữa sinh viên nam và nữ, sinh viên năm thứ nhất và năm thứ tư, sinh viên theo học khối ngành kỹ thuật và khối ngành kinh tế. Effect size (hệ số d) cũng được tính toán để xác định độ lớn của sự khác biệt.

Giới tính: Trong số 10 chỉ báo, sự khác biệt chỉ được ghi nhận ở Lập luận định lượng $(\mathrm{t}=2,02 ; \mathrm{d}$ $=0,19 ; \mathrm{p}<0,05)$ và Tương tác giữa sinh viên và giảng viên $(\mathrm{t}=2,08 ; \mathrm{d}=0,20 ; \mathrm{p}<0,05)$. Các sinh viên nam sử dụng các hoạt động lập luận định lượng và tương tác với giảng viên nhiều hơn sinh viên nữ nhưng mức độ khác biệt khá thấp, không đáng kể.

Năm hoc: Dữ liệu cho thấy không có nhiều sự khác biệt giữa sinh viên năm thứ nhất và năm thứ tư. Trong khi sinh viên năm thứ tư thực hiện các hoạt động lập luận định lượng $(\mathrm{t}=-2,76 ; \mathrm{d}=0,25 ; \mathrm{p}<$ $0,01)$ và học tập thông qua tích hợp và chiêm nghiệm $(\mathrm{t}=-2,65 ; \mathrm{d}=0,24 ; \mathrm{p}<0,01)$ thường xuyên hơn sinh viên năm thứ nhất, sinh viên năm thứ nhất lại cho rằng họ nhận được sự hỗ trợ của nhà trường nhiều hơn sinh viên năm thứ tư $(\mathrm{t}=2,00 ; \mathrm{d}=0,18 ; \mathrm{p}<0,05)$. Effect size $(\mathrm{d})$ cho thấy sự khác biệt này không lớn. Mức độ thực hiện các hoạt động lập luận định lượng và học tập thông qua tích hợp và chiêm nghiệm thường xuyên hơn ở sinh viên năm thứ tư có thể do trong giai đoạn này nhiều sinh viên phải thực hiện các đồ án, hay luận văn tốt nghiệp. Các nhiệm vụ này đòi hỏi sinh viên phải thường xuyên thực hiện các hoạt động trên. Sự khác biệt trong đánh giá của sinh viên về các hoạt động hỗ trợ của nhà trường cho thấy nhà trường đã quan tâm hơn và đã thực hiện một số cải thiện trong công tác hỗ trợ sinh viên. Điều đáng ngạc nhiên là nỗ lực học tập của sinh viên năm thứ tư không khác biệt so với sinh viên năm thứ nhất, cả về thời gian sinh viên dành cho việc học tập lẫn sự cố gắng trong học tập để hoàn thành các yêu cầu của giảng viên, của môn học. Cần có thêm các nghiên cứu để giải thích hiện tượng này.

Ngành hoc: Không tìm thấy bất kỳ sự khác biệt nào trong các chỉ báo giữa sinh viên học khối ngành kỹ thuật và sinh viên học khối ngành kinh tế.

Bảng 6: Sự khác biệt trong các chỉ báo giữa các nhóm sinh viên

\begin{tabular}{ccccc}
\hline Đặc điểm & \multicolumn{3}{c}{ Các chỉ báo } \\
\hline \multirow{3}{*}{ Giới tính } & LLĐL & TH\&CN & TTGV-SV & HTr \\
& $\mathrm{t}=2,02^{*}$ & & $\mathrm{t}=2,08$ & \\
Năm học & $\mathrm{d}=0,20$ & & $\mathrm{~d}=0,20^{*}$ & \\
& $\mathrm{t}=-2,76^{* *}$ & $\mathrm{t}=-2,65$ & & $\mathrm{t}=2,00$ \\
& $\mathrm{~d}=0,25$ & $\mathrm{~d}=0,24^{*}$ & & $\mathrm{~d}=0,24^{* *}$
\end{tabular}

Chú thích: $T H \& C N=H o c$ tập thông qua tích hợp và chiêm nghiệm; LLĐL = Lập luận định luợng; TTGV-SV= Tương tác giữa sinh viên và giảng viên; Htr = Sụ hố trợ của nhà trường.

$*=p<0,05 ; * *=p<0,01$

\section{KẾT LUẬN VÀ KHUYẾN NGH!}

Nghiên cứu này cho thấy sinh viên trường Đại học Công nghiệp TP. HCM chưa dành đủ thời gian và công sức cho việc học tập. Nguyên nhân dẫn đến tính trạng này có thể là do cách kiểm tra, đánh giá hiện nay đang đặt nặng vào các kỳ thi và thách thức học thuật chưa đủ cao để tạo áp lực cho sinh viên phải nỗ lực học tập. Đánh giá của sinh viên về các hoạt động giảng dạy và hỗ trợ của nhà trường có cả những khía cạnh tích cực và những khía cạnh cần cải thiện. Sinh viên đánh giá cao hiệu quả giảng dạy của giảng viên, và sự hỗ trợ của nhà trường dành cho họ trong học tập và trong cuộc sống. Nhà trường cũng tạo cơ hội cho sinh viên giao tiếp với nhau và thường xuyên hợp tác với nhau trong học tập. Bên cạnh những điểm tích cực này, dữ liệu cũng cho thấy chương trình học của nhà trường chưa chú trọng nhiều vào việc phát triển các kỹ năng tư duy bậc cao, khả năng tích hợp, chiêm nghiệm các nguồn kiến thức của sinh viên. Sinh viên cũng ít có cơ hội để tương tác với giảng viên. Sinh viên cũng không đánh 
giá tích cực mối quan hệ giữa họ với giảng viên, cố vấn học tập và các nhân viên phòng ban. Khi so sánh NLHT và các HĐGD\&HT của nhà trường giữa các nhóm sinh viên (giới tính, năm học, và ngành học) với nhau, chỉ có $4 / 10$ chỉ báo cho thấy có sự khác biệt nhưng mức độ khác biệt này không đáng kể về mặt thống kê.

Nghiên cứu cũng cho thấy một số ảnh hưởng của văn hóa đối với NLHT của sinh viên và các HĐGD\&HT của nhà trường. Ví dụ, do ảnh hưởng của đạo Khổng, sinh viên Việt Nam cũng như sinh viên Trung Quốc, Hàn Quốc ... tương tác với giảng viên ít hơn nhiều so với sinh viên Mỹ. Ảnh hưởng của đạo Khổng còn được tìm thấy qua cách giảng dạy chú trọng nhiều đến việc truyền thụ kiến thức có phần thụ động và việc kiểm tra đánh giá chủ yếu đặt nặng ở các kỳ thi ở cả Việt Nam và Trung Quốc. Không chỉ sinh viên Việt Nam chỉ tập trung học vào trước các kỳ thi, hiện tượng này cũng được phản ánh trong một số nghiên cứu về NLHT của sinh viên Trung Quốc. Điều này còn được thể hiện qua chương trình học ít hướng đến các hoạt động tư duy bậc cao hay học tập thông qua tích hợp và chiêm nghiệm ở cả hai nước.

Để thúc đẩy sinh viên nỗ lực hơn trong học tập, trước hết, nhà trường nên thay đổi cách kiểm tra đánh giá. Nhà trường nên tăng tỷ trọng điểm đánh giá quá trình. Sinh viên cần được giao nhiều bài tập, bài kiểm tra hơn. Khi đó, sinh viên sẽ phải tập trung học tập trong suốt học kỳ chứ không phải chỉ học trước các kỳ thi. Việc tăng số lượng bài kiểm tra, đánh giá có thể làm tăng khối lượng công việc của giảng viên. Nhà trường có thể giải quyết vấn đề này thông qua việc sử dụng các hệ thống quản lý học tập online hoặc thuê các trợ giảng hỗ trợ giảng viên trong hướng dẫn, kiểm tra và chẩm bài tập trên lớp và về nhà của sinh viên. Bên cạnh đó, nhà trường cần đưa thêm vào chương trình học các nội dung hướng đến việc phát triển tư duy bậc cao của sinh viên hay khuyến khích sinh viên học tập thông qua tích hợp và chiêm nghiệm. Những hoạt động này sẽ tạo áp lực học tập cho sinh viên buộc sinh viên phải đọc tài liệu, tìm kiếm thông tin nhiều hơn, tương tác nhiều hơn với giảng viên và bạn học, phải tư duy nhiều hơn. Nhờ vậy, sinh viên sẽ tích lũy được thêm nhiều kiến thức và phát huy được năng lực của bản thân. Ngoài ra, nhà trường cần cải thiện mối quan hệ giữa sinh viên với giảng viên, cố vấn học tập và nhân viên phòng ban. Các hoạt động giảng dạy, hỗ trợ sinh viên nên được đặt trên cơ sở lấy sinh viên làm trung tâm để tạo ra một môi trường học tập thân thiện với sinh viên. Khi nhà trường thúc đẩy được sinh viên nỗ lực học tập, tạo ra được môi trường thuận lợi cho sinh viên học tập, nhà trường sẽ có thể nâng cao được chất lượng đào tạo của mình.

\section{LỜI CẢM ƠN}

Nhóm tác giả chân thành cảm ơn Trường Đại học Công nghiệp TP.HCM đã cấp kinh phí để thực hiện đề tài nghiên cứu này theo Hợp đồng nghiên cứu khoa học số 18/HĐ-ĐHCN, mã số 183.CB01.

\section{TÀI LIỆU THAM KHẢO}

[1] Kuh, G.D., 2009. The National Survey of Student Engagement: Conceptual and empirical foundations, New Directions for Institutional Research, 5-20 (141), pp. 5-20.

[2] Trowler, V., 2010. Student engagement literature review, York: Higher Education Academy. Available at $\langle$ https://www.heacademy.ac.uk/sites/default/files/studentengagementliteraturereview.pdf>.

[3] Coates, H., 2005. The value of student engagement for higher education quality assurance, Quality in Higher Education, 11 (1), pp. 25-36.

[4] Tran, T.T., 2014. Is graduate employability the 'whole-of-higher-education-issue?, Journal of Education and Work, pp. 1-21.

[5] Tran, T.T, 2013. Limitation on the development of skills in higher education in Vietnam, Higher Education: The International Journal of Higher Education and Educational Planning, 65 (5), pp. 631-644.

[6] Viện Ngôn Ngữ Học, Hoàng Phê (chủ biên), 2003. Tù điển tiếng Việt. In lần thứ 9, Hà Nội - Đà Nẵng: NXB Đà Nẵng.

[7] Zhao, C-M \& Kuh, GD 2004, 'Adding value: learning communities and student engagement', Research in

Higher Education, 45 (2), pp. 115-138.

[8] Lê Văn Lập, 2011. Phuơng pháp giảng dạy và tâm lý giáo dục trong truờng học-Kỹ năng quản lý và giảng dạy đạt hiệu quả cao, NXB Lao động. 
[9] Hoàng Thị Thu Hiền \& Nguyễn Thị Lan, 2012 Giáo trìnhTâm lý học, TP. HCM: NXB Đại học Quốc gia TP.HCM.

[10] Đoàn Huy Oánh, 2005. Tâm lý sue phạm, TP.HCM: NXB Đại học Quốc gia TP.HCM.

[11] Nguyễn Công Khanh, 2008. Nghiên cứu phong cách học của sinh viên, Tạp chí Giáo dục, 202, tr. 7-10.

[12] Kuh, G.D., Kinzie, J., Buckley, J.A., Bridges, B.K. \& Hayek, J.C., 2007. Piecing together the student success puzzle: research, propositions, and recommendations, ASHE Higher Education Report, 32 (5), pp. 1-182.

[13] National Survey of Student Engagement (NSSE), 2013. Engagement indicators, NSSE, IN: Indiana University Center for Postsecondary Research, Bloomington, Available at <http://nsse.indiana.edu/html/engagement_indicators.cfm>.

[14] Krause, K-L. \& Coates, H., 2008. Students' engagement in first-year university, Assessment \& Evaluation in Higher Education, 33 (5), pp. 493-505.

[15] Radloff, A. \& Coates, H., 2010. Doing more for learning: enhancing engagement and outcomes. Australasian Student Engagement Report, Camberwell: Australian Council for Educational Research (ACER. Available at $<$ http://research.acer.edu.au/ausse/12/>

[16] Pascarella, ET \& Terenzini, P.T., 2005. How college affects students : a third decade of research, 2nd edn, San Francisco: Jossey-Bass.

[17] Coates, H., 2008. Beyond happiness: managing engagement to enhance satisfaction and grades, Camberwell: Australian Council for Educational Research (ACER).

[18] Carini, R.M., Kuh, G.D. \& Klein, S.P., 2006. Student engagement and student learning: Testing the linkage, Research in Higher Education, 47 (1), pp. 1-32.

[19] Kuh, G.D., Cruce, T.M., Shoup, R., Kinzie, J. \& Gonyea, R.M., 2008. Unmasking the effects of student engagement on first-year college grades and persistence, The Journal of Higher Education, 79 (5), pp. 540-563.

[20] Pike, G.R., Kuh, G.D., McCormick, A.C., Ethington, C.A. \& Smart, J.C., 2011. If and when money matters: the relationships among educational expenditures, student engagement and students' learning outcomes, Research in Higher Education, 52 (1), pp. 81-106.

[21] Gordon, J., Ludlum, J.\& Hoey, J.J., 2008.Validating NSSE against Student Outcomes: Are They Related?, Research in Higher Education, 49 (1), pp. 19-39.

[22] National Survey of Student Engagement (NSSE), 2018. NSSE 2018 Engagement indicators, NSSE, IN: Indiana University Center for Postsecondary Research, Bloomington, Available at <http://nsse.indiana.edu/2018_Institutional_Report/pdf/NSSE18\%20Engagement\%20Indicators\%20(NSSEville $\% 20$ State).pdf>

[23] Kuh, G.D., Kinzie, J., Schuh, J.H., Whitt, E.J. \& Associates, 2005. Student success in college: Creating conditions that matter, 1st edn, San Francisco: Jossey-Bass.

[24] Nguyen, T.T.T, 2016. Student engagement: A useful quality concept in the Vietnamese Higher Education (PhD Thesis).

[25] Ko, J., Park, S., Yu, H., Kim, S-J. \& Kim, D., 2016. 'The Structural Relationship Between Student Engagement and Learning Outcomes in Korea', The Asia-Pacific Education Researcher, 25 (1), pp. 147-157.

[26] Luo, Y., 2015. Learning ants: a portrait of Chinese college students in mass higher education, in JC Shin, GA Postiglione \& F Huang (eds), Mass higher education development in East Asia: strategy, quality, and challenges, 2, Springer, pp. 177 - 188.

[27] Zhang, Z., Hu, W. \& McNamara, O., 2015. Undergraduate student engagement at a Chinese university: a case study, Educational Assessment, Evaluation and Accountability, 27 (2), pp. 105-127.

Ngày nhận bài: 13/01/2018

Ngày chấp nhận đăng:02/02/2018 